\title{
Diseño y construcción de un
}

analizador de redes eléctricas para sistemas trifásicos de bajo voltaje

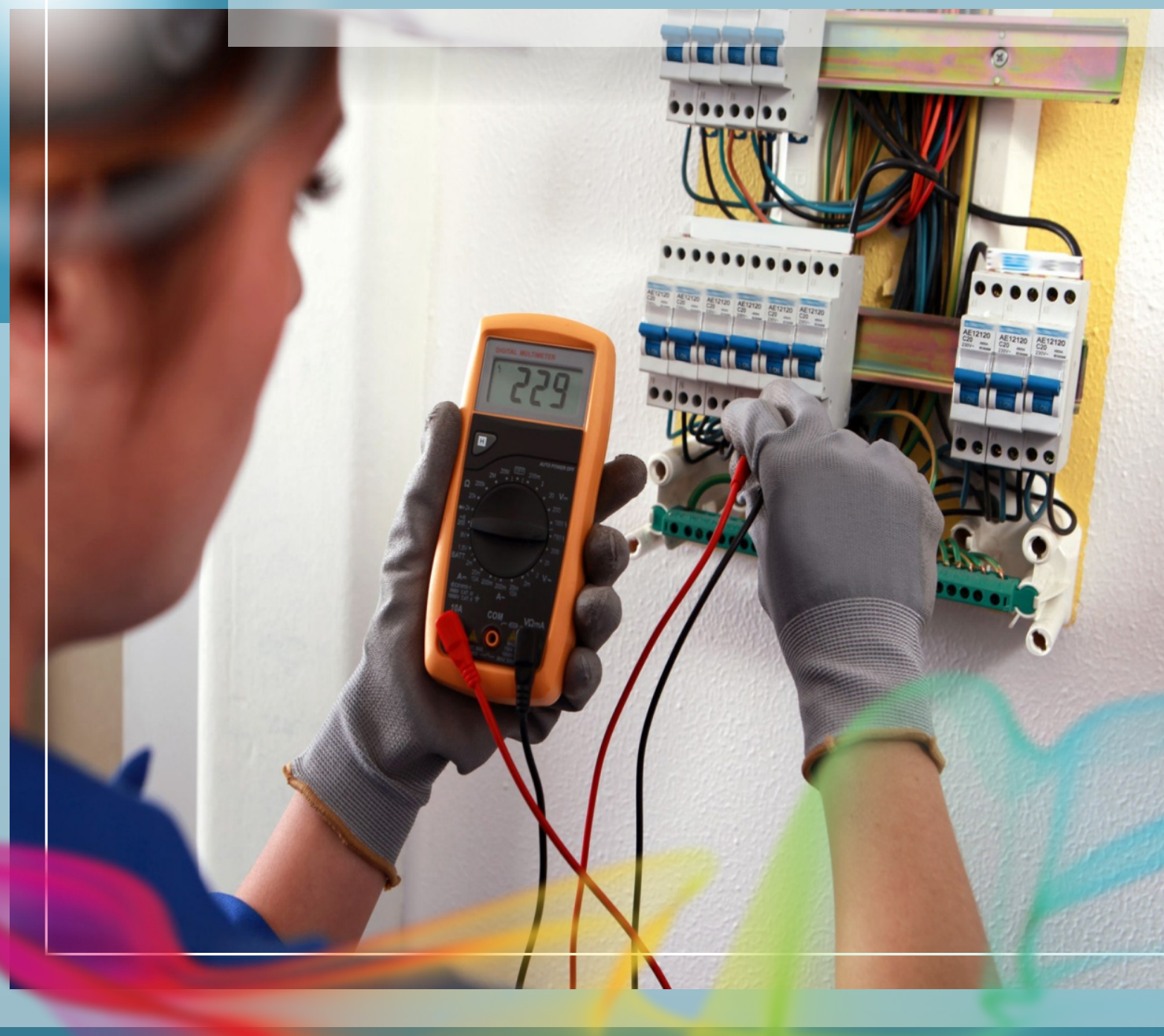




\title{
Diseño y construcción de un analizador de redes eléctricas para sistemas trifásicos de bajo voltaje ${ }^{1}$. Design and Construction of a Quality Analyzer Electricity Networks for Low Voltage Three-Phase Systems.
}

\author{
Iván Fernando Rincón Roa ${ }^{2}$, William Gustavo Cadena Téllez ${ }^{3}$, \\ Marco Fidel Flórez Franco ${ }^{4}$. \\ ${ }^{1}$ Unidades Tecnológicas de Santander (UTS) Bucaramanga, Colombia.
}

Artículo recibido en noviembre 2015; artículo aceptado en noviembre de 2015.

Citación del artículo: Rincón, I., Cadena, W. \& Flórez, M. (2015). Diseño y construcción de un analizador de redes eléctricas para sistemas trifásicos de bajo voltaje. I+D Revista de Investigaciones, $6(2), 57-75$.

\section{Resumen}

El analizador de redes de calidad es un dispositivo electrónico portátil que ayuda a solucionar los problemas en los sistemas de distribución de energía de forma rápida, segura y detallada. Este instrumento portátil tiene un diseño que utiliza tecnología avanzada para los circuitos integrados y procesadores de señales digitales, componentes de montaje superficial a nivel profesional, una interfaz de usuario fácil de usar y la vivienda adecuada para el uso de la herramienta.

Este instrumento fue desarrollado para medir señales eléctricas de tensión y corriente en tiempo real, utilizando cuatro cables de prueba y pinzas de cocodrilo 3 HT 4003 CA transductores pinzas conectadas a la parte posterior de la unidad. Este prototipo permite la visualización de las curvas y datos numéricos de tres tensiones de fase, tres tensiones de línea, tres corrientes, el

\footnotetext{
${ }^{1}$ Artículo de investigación, resultado de un proyecto de investigación culminado, perteneciente al área de electrónica, desarrollado y financiado por las Unidades Tecnológicas de Santander (UTS) de la ciudad de Bucaramanga (Colombia). Dirección: Calle de los Estudiantes No. 9-82 Ciudadela Real de Minas, PBX:6917700. Fecha de inicio: Enero de 2014, fecha de terminación: junio de 2015.

${ }^{2}$ Ingeniero Electrónico, Unidades Tecnológicas de Santander, Investigador del CEAC de las Unidades Tecnológicas de Santander. Dirección: Calle de los Estudiantes No. 9-82 Ciudadela Real de Minas, PBX:6917700.

${ }^{3}$ Ingeniero Electrónico, Unidades Tecnológicas de Santander, Investigador del CEAC de las Unidades Tecnológicas de Santander. Dirección: Calle de los Estudiantes No. 9-82 Ciudadela Real de Minas, PBX:6917700.

${ }^{4}$ Ingeniero electricista, Universidad Industrial de Santander. Especialista en Telecomunicaciones, Universidad Pontificia Bolivariana de Bucaramanga. Magíster en Ingeniería, área de Telecomunicaciones, Universidad Pontificia Bolivariana sede Medellín, docente investigador de las Unidades Tecnológicas de Santander, vinculado como director del grupo de investigación CEAC (Conversión de Energía Automática y Control). Su campo de investigación se centra en el procesamiento digital de señales y generación de equipo biomédico.
} 
cambio de fase entre las señales de tensión y señales de corriente entre las señales de voltaje contra el cálculo de la potencia activa, potencia reactiva, complejo y factor de medición de armónicos y el cálculo del THD de cada una de las señales, y la medición de frecuencia para las señales de referencia.

Palabras clave: analizador de redes, calidad de la energía, sistemas trifásicos, armónicos, dsPIC, pantalla táctil.

\section{Abstract}

The quality analyzer grids is a portable electronic device that helps troubleshoot problems in power distribution systems quickly, securely and detailed. This portable instrument has a design that uses advanced technology for integrated circuits and digital signal processors surface mount components professional level, a user interface easy to use and housing suitable for the use of the tool.

This instrument was developed to measure electrical signals of voltage and current in real time using $4+$ test leads alligator clips and $3 \mathrm{HT}$ 4003 AC transducers clamps connected to the back of the unit. This prototype allows the display of curves and numerical data of 3 phase voltages, three line voltages, 3 currents, the phase shift between the signals of voltage and current signals between vs. voltage signals, the calculation of active power, reactive, complex and power factor, harmonics measurement and calculation of the THD of each of the signals, and the frequency measurement for the reference signals.
Keywords: network analyzer, power quality, three-phase systems, harmonics, dsPIC, touch screen.

\section{Introducción}

La existencia de armónicos en las redes conduce a problemas en el funcionamiento de estas, como sobrecalentamientos en los conductores, disparos intempestivos de interruptores automáticos y diferenciales, disminución del factor de potencia, deterioro de la forma de onda de la tensión entre otras (Zhou, 2012). Por tal motivo, la calidad de la red eléctrica es fundamental para mantener el servicio en sectores en los que los equipos eléctricos y electrónicos sean indispensables y las cargas no lineales, pues las conmutaciones, las maniobras de cambio de carga o las propias averías de los equipos pueden ocasionar una mala calidad en el suministro eléctrico (Herrera \& Flórez, 2004).

De acuerdo a lo anterior, la finalidad de este trabajo es presentar una alternativa en hardware aplicando los controladores digitales de señal dsPIC para la implementación de los algoritmos de medición y cálculo de las señales eléctricas, con el fin de monitorizar en tiempo real dichas señales, presentes en las redes de los sistemas trifásicos y así poder tomar medidas de precaución y corrección que eviten daños mayores; además, el uso de una pantalla Touch Screen a color de 3,2 pulgadas permite la visualización de señales y datos e interfaz con el usuario. El equipo final es de bajo costo y podría ser adquirido para la vigilancia de redes en la mediana industria colombiana. 
Este nuevo equipo permite visualizar parámetros de las señales eléctricas con un error máximo en la exactitud del $4 \%$ en las medidas de corriente, voltaje, frecuencia, distorsión armónica, potencia y desfases entre las señales, con un mínimo de hardware, con costos de elaboración y consumo muy bajos, y brindando muy buenas prestaciones, además de su confiabilidad.

\section{Metodología y desarrollo}

\section{Tipo de estudio}

La investigación realizada fue del tipo aplicada y su principal objetivo es el desarrollo de un equipo económico que permita el análisis de la calidad del servicio de energía eléctrica trifásica de baja tensión.

\section{Tarjeta de desarrollo}

El prototipo del analizador es un sistema de adquisición de señales eléctricas a las cuales se les hace una adecuación y un tratamiento para su respectiva medición y cálculo (Gómez, 2011). Dicho equipo cuenta con una tarjeta electrónica de desarrollo que está compuesta por una serie de etapas, que son: fuente de alimentación, divisor de tensión, protección contra sobre picos de tensión, adecuación de señales de voltaje $\mathrm{y}$ corriente, filtrado, procesamiento de señales, control de la pantalla y visualización.

Además, se implementó el diseño de una tarjeta de desarrollo para una pantalla touchscreen de 3,2 pulgadas, modelo TFT2N0369-E, de tal manera que el usuario pueda visualizar y manipular el equipo de una forma más adecuada y sencilla.

La tarjeta de adquisición de señales eléctricas trifásicas (Carrasco J. , s.f.), de voltaje y corriente acondiciona las señales censadas por los transductores tipo pinza y por las puntas de prueba tipo cocodrilo (HT Instruments, s.f.), hasta llegar a la entrada del ADC del dsPIC, el cual le envía, por medio de un protocolo de comunicación SPI, los datos medidos y calculados al dsPIC que controla la TFT-LCD (Escobar \& Jácome, 2009), este, a su vez, envía los datos que fueron solicitados por el usuario a la pantalla touchscreen para su respectiva visualización; en la pantalla touchscreen el usuario podrá monitorizar y ver los datos de sus mediciones en tiempo real. 
Figura 1. Diagrama de bloques de la tarjeta de desarrollo del analizador

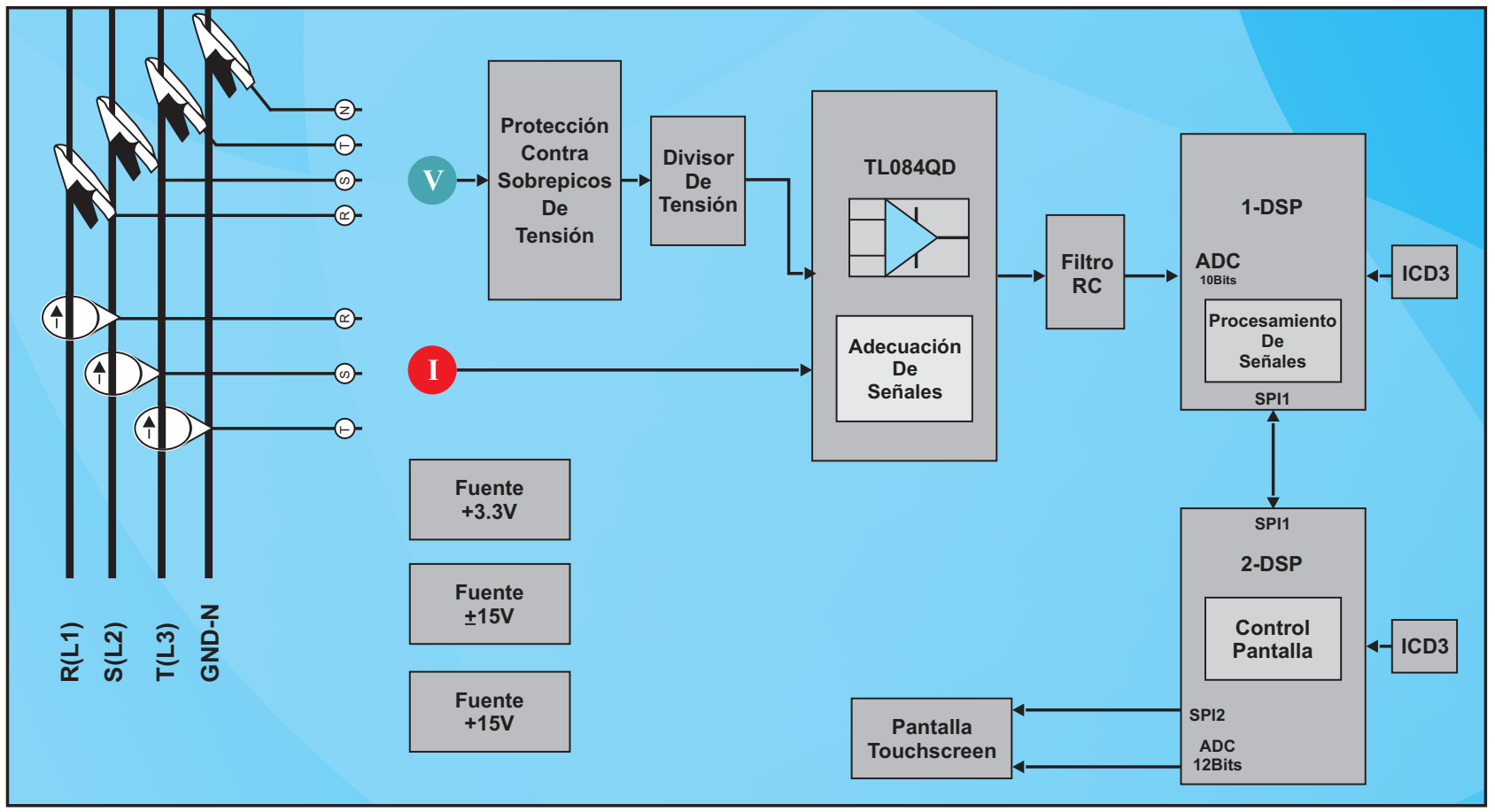

Fuente: Los autores.

A continuación se abordan cada una de las etapas de la tarjeta de desarrollo.

\section{Etapa de la fuente de alimentación}

La etapa de alimentación se diseñó teniendo en cuenta la alimentación requerida por los elementos a usar, una serie de arreglos de reguladores que suministran el voltaje a los dispositivos analógicos y digitales.

La fuente de alimentación se divide en dos secciones: la sección $\mathrm{A}$ es un diseño de adecuación y protección del suministro de voltaje DC; la parte B está compuesta por tres circuitos integrados reguladores de voltaje fijo positivos que son: LM2937IMPX-15 (National Semicondutor Corporation. LM2937IMPX-15 Datasheet., s.f.), UA78M08CKVURG3 (Texas Instruments Inc. UA78M33C Datasheet, 2012),
UA78M33CKVURG3 (Texas Instruments Inc. UA78M08C Datasheet., 2012), los cuales entregan a la salida $+15 \mathrm{~V},+8 \mathrm{~V}$ y $+3.3 \mathrm{~V}$ respectivamente, con una corriente de salida máxima de $500 \mathrm{~mA}$, un conversor DC-DC VFA1205MP-6W que entrega a su salida un voltaje dual de $\pm 5 \mathrm{~V}$, con una corriente máxima de salida de $600 \mathrm{~mA}$ en cada fuente, y una serie de elementos pasivos como resistencias, condensadores y bobinas, cuyos valores son los recomendados por los fabricantes e implementados para la protección y adecuado funcionamiento del equipo.

\section{Etapa de protección contra sobrepicos de tensión de AC}

Antes de la entrada de la etapa de adquisición y adecuación de las señales eléctricas se incluyen circuitos de filtrado y protección adicional en el 
diseño del medidor. Las Ferrite beads 1,2, 3 son los núcleos de ferrita integrados de $800 \mathrm{~mA} / 150$ OHM, C 60, 61, 62 son condensadores de $0.1 \mathrm{uF} / 305 \mathrm{VAC}$ y Varistor 1, 2, 3 son varistores de 275 Vrms de alta tensión que se utilizan para mejorar el rendimiento contra sobretensiones del sistema.

Para evitar que las señales de las corrientes alternas de alta frecuencia que circulan por los cables se expandan hacia el interior como señales ruidosas de radiofrecuencia o ruidos parásitos, se colocan los núcleos de ferrita en la entrada. De esa forma, el tubito de ferrita se convierte en un "filtro inductor de alta reactancia o alta resistencia" (también denominado "choque"), cuya función principal es ofrecer gran oposición al paso y propagación interna de determinadas altas frecuencias de corrientes alternas que se circulan por los cables(Mornsun, 2012).

El principio de funcionamiento de este tipo de filtro con alta reactancia radica en que, cuando las corrientes alternas de alta frecuencia tratan de atravesar la parte del cable donde se encuentra ubicado el "choque" o núcleo de ferrita, la autoinductancia que produce el flujo magnético de la corriente que trata de atravesarlo genera una fuerza contra electromotriz que se opone al paso de su componente alterna.

Sin embargo, este filtro no impide que la corriente directa (C.D.) o continua (C.C.), ni la corriente alterna de baja frecuencia lo atraviesen y puedan continuar fluyendo libremente por todo el cable y el resto del circuito electrónico.

Los varistores y los condensadores, que se ponen en paralelo al circuito a proteger, absorben todos los picos mayores a su tensión nominal. El varistor solo suprime picos transitorios, y si es sometido a una tensión elevada constante, se destruye(Microchip Inc, 2009).

\section{Etapa divisor de tensión}

Esta etapa muestra un esquema básico de un divisor de voltaje alterno, como su nombre lo indica; el divisor se compone de tres resistencias, dos resistencias superficiales, una de $1 \mathrm{M} \Omega$ $1 / 2 \mathrm{~W}$ a $1 \%$ de tolerancia, una de $900 \mathrm{k} \Omega-1 / 2 \mathrm{~W}$ a $1 \%$ de tolerancia y una resistencia variable de precisión, conocida como trimmer, de $20 \mathrm{k} \Omega$, las cuales están conectadas en serie de tal forma que la tensión de entrada V_R, V_S y V_T $(0 \mathrm{~V}-$ 220Vrms) -al igual que la potencia- se reduzca en cada una de las resistencias; la tensión de salida Vout se obtiene del punto medio entre dos resistencias y tiene un valor mínimo de $0 \mathrm{~V}$ y un valor máximo de $3.3 \mathrm{Vp}-\mathrm{p}$, requerimientos necesarios para trabajar con el ADC del dsPIC.

Esta fase es de vital importancia, porque en la etapa de adecuación y adquisición de las señales no se puede trabajar con valores tan altos en las señales eléctricas ya que se dañarían los OPAMPs, los DSPs y los elementos pasivos que sobrepasen sus niveles de voltaje y potencia nominales.

Los valores máximos de potencia medidos en cada uno de los elementos de esta etapa son de: $\mathrm{Pt} \mathrm{R}$ e s $(1 \mathrm{M} \Omega)=13.266 \mathrm{~m} \mathrm{~W}$, P t R e s $\left(\begin{array}{llll}9 & 0 & 0 & \mathrm{~K} \Omega\end{array}\right)=11.938 \mathrm{~m} \mathrm{~W}$, $\operatorname{PtRes}(20 \mathrm{k} \Omega)=133.981 \mathrm{uW}$ con una tensión máxima de entrada de $220 \mathrm{Vrms}$.

\section{Etapa de adecuación de las señales}

A las señales sensadas por los transductores 
tipo pinza y las señales que salen de la etapa del divisor de tensión se les debe sumar una señal de offset que ubique el pico más negativo de la señal por encima de cero, asimismo el pico más positivo debe ser de $3,3 \mathrm{~V}$, para lo cual a $220 \mathrm{Vrms}$ y 150Arms se tienen 3.3V (1Arms y 150Arms son los valores mínimo y máximo de corriente que debe medir la pinza para conservar intactas sus características de medición; la medición de voltaje estará en el rango de 0V - 220Vrms); por lo tanto, el cero de la señal análoga estará ubicado en $1.65 \mathrm{~V}$ y $\sin$ importar la amplitud de las señales siempre estará sobre este nivel de offset.

Para realizar esta tarea se realizaron dos diseños, uno para adecuar la señal de voltaje proveniente del transductor tipo pinza y otra para adecuar la señal de voltaje que sale de la etapa del divisor de tensión, dichas etapas funcionan de la siguiente manera:

Para cada fase de voltaje se implementó un amplificador sumador no inversor acoplado en alterna con ganancia de 1, un amplificador sumador no inversor acoplado en alterna con ganancia de 2 y un seguidor de tensión que tiene fijado en salida 1,65V (offset positivo) que es el nivel de la señal de continua que se sumará a la tensión de alterna mediante el IC TL084QD (Texas Instruments Inc., 2012), y resistencias. La figura 2 muestra la señal de entrada (Senoidal Amarilla) a la etapa de adecuación de señales con respecto a la señal de salida (Senoidal Azul) ya con el offset de $1.65 \mathrm{~V}$ y una amplitud máxima de $3.3 \mathrm{Vp}-\mathrm{p}$, estando la señal sujetada sobre cero.

Para cada fase de corriente se implementó un amplificador inversor con ganancia de 7.8 para amplificar la señal proveniente de los transductores tipo pinza que tienen un rango da salida de tensión de $1 \mathrm{mV}-150 \mathrm{mV}$ (para $1 \mathrm{Amp}-$ 150Amp) para que quedara de $11 \mathrm{mV}-3.3 \mathrm{~V}$ (rango de valores de entrada permitidos en el conversor del dsPIC) (Microchip Inc, s.f.), un amplificador sumador no inversor acoplado en alterna con ganancia de 2 y un seguidor de tensión que tiene fijado en salida 1,65V (offset positivo) que es el nivel de la señal de continua que se sumará a la tensión de alterna mediante el IC TL084QD y resistencias.

Figura 2. Señal de entrada contra señal de salida en la etapa de adecuación de voltaje simulada en NI Multisim

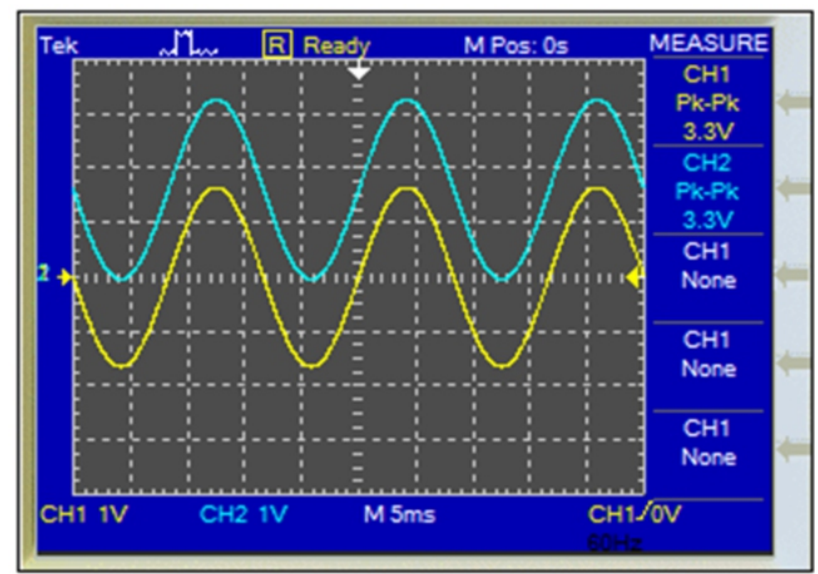

Fuente: Los autores

\section{Etapa de filtrado}

El filtro en la tarjeta de desarrollo se implementó con el fin de restringir el paso de frecuencias no deseadas, es decir frecuencias por encima del último armónico a evaluar; a esta determinada frecuencia se le denomina frecuencia de corte y sirve para disminuir el tiempo de procesamiento y la aparición de frecuencias redundantes. Se dice que a esa frecuencia de corte la amplitud de la señal de salida del filtro reduce su valor. 
Al Vin llegan cada una de las señales de la etapa de adecuación de señales de voltaje y corriente y de Vout salen las señales que llegan al ADC del dsPIC para su respectivo procesamiento. Se utilizó un filtro pasivo $\mathrm{RC}$ pasa bajas, con una frecuencia de corte de $3500 \mathrm{~Hz}$.

\section{Etapa de procesamiento de señales}

En esta etapa se implementó el dsPIC (Microchip Inc, s.f.), el cual se usa para tratar las señales de voltaje y corriente. Es un procesador de señales digitales muy rápido y poderoso, capaz de computar señales en tiempo real, debido a esto es especialmente útil para el procesamiento y representación de señales analógicas en tiempo real. En un sistema en tiempo real se reciben muestras normalmente provenientes de un conversor analógico/digital (ADC), el dsPIC tiene un hardware y un software optimizados para este trabajo.

Específicamente, las funciones que cumple el dsPIC para este proyecto en la tarjeta de desarrollo son: la medición y el cálculo de las señales de voltajes y corrientes de línea y de fase, frecuencia, THD, potencia activa, reactiva, compleja, y desfases entre las señales, y envío y recepción de datos por medio del módulo de comunicación SPI1 al dsPIC.

La adquisición de los datos se hace por medio del ADC del dsPIC; cada una de las seis señales llega a los puertos de RB0 a RB5, que son los canales de entrada análogos del ADC, AN0 a AN5. Adicionalmente, se integró el circuito de programación del dsPIC compatible con el programador PICkitTM 3 de Microchip.

\section{Etapa control de la pantalla}

Esta etapa fue diseñada con otro dsPIC para el control y el manejo de la pantalla touchscreen TFT2N0369-E y así poder hacer más rápido el procesamiento del dsPIC.

Específicamente, las funciones que cumple el dsPIC son: comunicación (envío y recepción de datos) entre los dos dsPIC por medio del módulo SPI1, control y diseño del menú grafico de la pantalla, manejo del sistema táctil y comunicación (envío y recepción de datos) entre el segundo dsPIC con la pantalla touchscreen por medio del módulo SPI2 para la visualización de las señales eléctricas y los datos numéricos.

\section{Etapa de visualización}

Para esta etapa se diseñó una tarjeta electrónica simple, donde se hallan los conectores de datos de 40 hilos y el conector de 4 hilos correspondiente a la interfaz touchscreen. Por medio de los jumpers PS2 y PS0 se seleccionó la interfaz SPI de 4 vías (SDI, SDO, SCK y CS). Los pines $\mathrm{X}+, \mathrm{X}-, \mathrm{Y}+$ y $\mathrm{Y}-$ de la pantalla se conectaron a los pines de entrada analógico/digital AN18, AN31, AN16 y AN30 del dsPIC(Rojas \& Reyes, 2011).

Figura 3. Coordenadas de los pixeles y del touch en el plano cartesiano de la pantalla touchscreen 


\section{TFT2N0369-E}

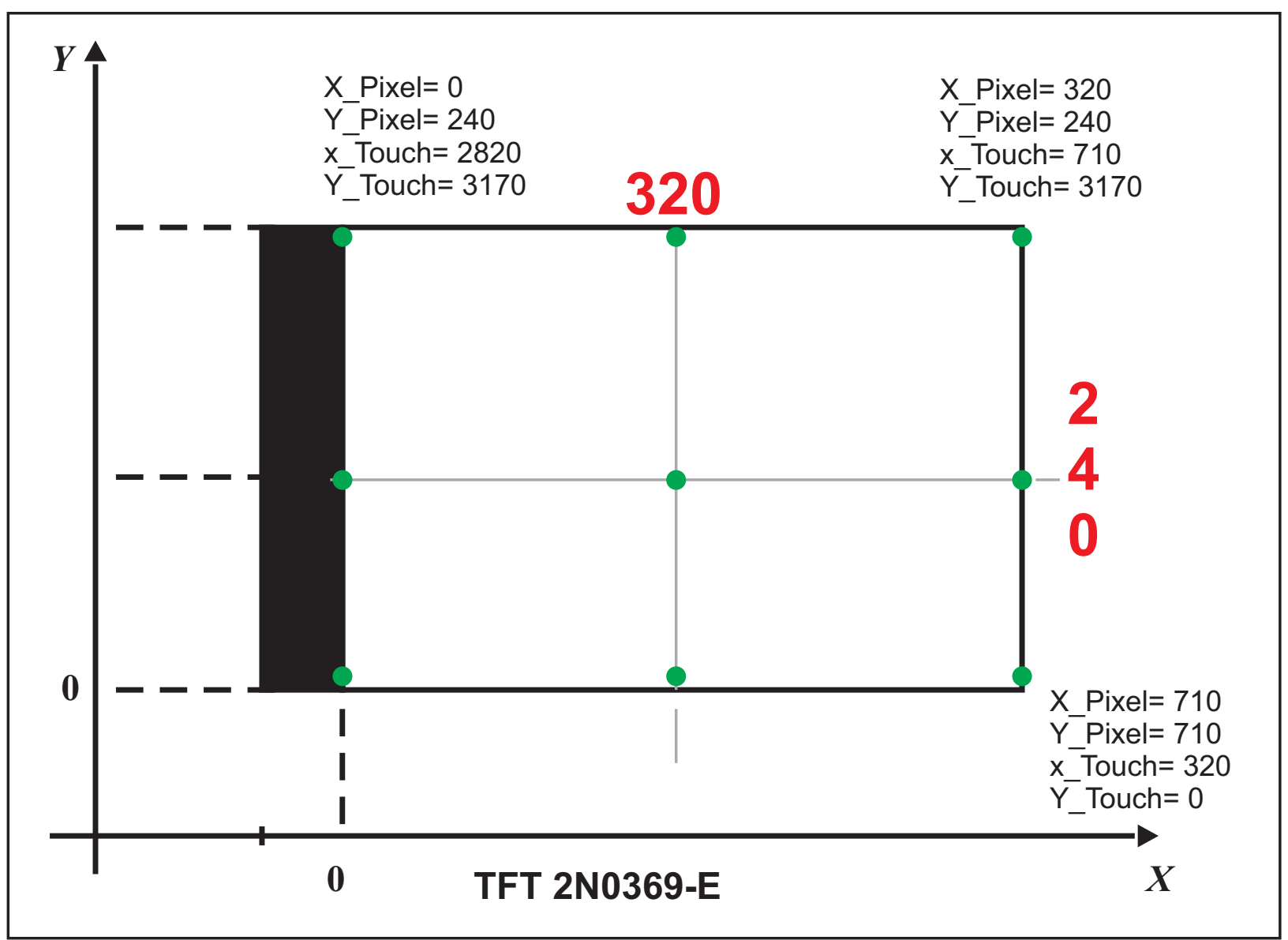

Fuente: Los autores.

\section{Software implementado}

Se desarrollaron dos tipos de software para dsPIC en lenguaje $\mathrm{C}$, utilizando el compilador C30 de Microchip (Clavijo, 2011). Se implementó un software para el dsPIC en el que se configuraron los módulos internos SPI, TIMER3 y ADC, y se desarrolló un código para la medición y cálculo de las señales eléctricas.

Para el dsPIC se implementó un software en el cual se incluyó la configuración de los módulos internos dentro de los que se destacan el SPI1,
SPI2 y ADC. Este software contiene la inicialización y configuración del periférico utilizado y la pantalla TFT2N0369-E. Para esta pantalla se desarrolló una interfaz de usuario para la configuración y visualización de datos y señales de eléctricas, además, un algoritmo que funciona como driver para el manejo del sistema táctil de la pantalla(Rojas \& Reyes, 2011).

Inicialmente, se configuró el reloj interno de los dsPICs utilizando los bits de control PLL, llevando los osciladores internos de $7.37 \mathrm{MHz}$ 
hasta $40 \mathrm{MHz}$, trabajando de esta forma a la máxima velocidad.

La configuración del dsPIC es la siguiente: se inicializó el módulo de comunicación SPI1 para la comunicación entre los DSPs, con una frecuencia de reloj de $5 \mathrm{MHz}$, y se configuró como maestro.

El módulo ADC1 se configuró a 10 bits para trabajar a una secuencia de conversión de muestreo multicanal, se habilitaron seis canales para escanear y tomar las lecturas de las señales eléctricas, desde AN0 a AN5; las entradas análogas pares se utilizaron para el voltaje y las entradas análogas impares se utilizaron para la corriente. El tamaño de los buffers de las entradas análogas es de 360; se toman 360 muestras por periodo para cada señal de entrada que permite la medición de voltaje, corriente, desfase y el cálculo de los armónicos de las señales.

El Tmr3 se configuró para trabajar a una frecuencia de muestreo de $21600 \mathrm{~Hz}$, la cual trabaja para la medición de voltajes, corrientes y desfases entre las señales, y la medición de los armónicos de las señales de voltaje y corriente, además de la medición de la frecuencia fundamental y el THD(Scribd., 2011).

La configuración del dsPIC es la siguiente: el módulo ADC1 se utilizó para realizar la conversión de las señales del sistema táctil de la pantalla y se configuró a una resolución de 12 bits. Se inicializaron los módulos de comunicación, el módulo SPI1 para la comunicación entre los dsPICs el cual se configuró como esclavo y se inicializó el módulo de comunicación SPI2 para la pantalla
TFT2N0369-E, con una frecuencia de reloj de 20 Mhz.

A través de la pantalla touchscreen se implementó una interfaz que permitirá al usuario la selección de los parámetros principales de las señales eléctricas provenientes de una red eléctrica monofásica o trifásica. La interfaz cuenta con tres diferentes entornos que cumplen con una función específica; estos son:

Pantalla inicial 1.

Pantalla inicial 2.

Pantallas de configuración y visualización.

\section{Algoritmo para la medición y el cálculo de señales eléctricas}

Para el cálculo de las señales eléctricas se implementó un algoritmo de medición de los voltajes de fase, corrientes de fase, el cálculo del desfase entre las señales y los voltajes de línea, adicionalmente permite la medición de las potencias activas, reactivas, complejas y el factor de potencia de una red trifásica o monofásica. Este algoritmo aplica a seis vectores de 360 muestras lo que corresponde al escaneo de seis canales del ADC1 de forma secuencial del dsPIC, el cual trabaja a una frecuencia de muestreo de $21600 \mathrm{~Hz}$.

Las señales eléctricas son medidas por los diferentes dispositivos como transductores de corriente tipo pinza de efecto Hall de referencia HT 4003, y las pinzas de voltaje tipo cocodrilo; las señales pasan por un tratamiento y adecuación para poderlas escanear y procesar en el dsPIC; para el escaneo y conversión del ADC desde AN0 a AN5 están habilitados seis canales, los canales pares son para las señales de voltaje y los impares para las señales de corriente; se toman 360 
muestras en cada canal y son almacenadas en seis buffers diferentes para realizar su respectivo cálculo.

Para el cálculo de los voltajes de fase y las corrientes de fase, los vectores de 360 muestras se pasan por una función que calcula el voltaje y la corriente RMS de dichos vectores y convierte esta información en un único dato, al cual se le aplican una serie de operaciones aritméticas y una linealización para obtener medidas más exactas; de los vectores de 360 muestras se toman 60 para obtener las curvas de las señales de voltajes y corrientes de fase.

Algo muy similar se hace con los voltajes de línea, con la diferencia que se deben restar cada una de las muestras de los buffers de las entradas análogas pares, después se hace el mismo procedimiento descrito en el párrafo anterior.

En cuanto al desfase, éste se calcula como el cambio de fase de las señales (de positivo a negativo), es también conocido como detector de cruce por cero, y se restan en cada una de las muestras en las cuales la señal cruzó el cero con respecto a la de los otros buffers; como la señal es de 360 muestras cada muestra equivale a un grado en el diagrama fasorial.

Para el cálculo de potencias de cada fase se implementaron las siguientes operaciones (Hayt \& Kemmerly, 2005):
Potencia activa o resistiva (real) $P$ :

$$
\mathrm{P}=\mathrm{V} * \mathrm{I} * \cos (\varphi)
$$

Ec. 1

Potencia reactiva o inductiva $Q$ :

$$
\mathrm{Q}=\mathrm{V} * \mathrm{I} * \sin (\varphi)
$$

Ec. 2

Potencia aparente o total (compleja) S:

$$
|\overline{\mathrm{S}}|=\mathrm{VI}
$$

Ec. 3

Factor de potencia FP:

$$
\mathrm{PF}=\cos (\varphi)
$$

Ec. 4

Dónde:

$V=$ Voltajes de fase

$I=$ Corrientes defase

$\varphi=$ Desfase

\section{Algoritmo para el cálculo de la DTF}

La transformada de Fourier implica múltiples cálculos matemáticos como sumas, multiplicaciones y evaluaciones trigonométricas. Estos procesos hacen lento el rendimiento de la máquina de proceso. Por esta razón la ciencia de tratamiento de señales se vio en la necesidad de buscar técnicas para realizar la transformada de Fourier de forma mucho más eficiente; de este esfuerzo surge como resultado notable el algoritmo de transformación rápida de Fourier, o FFT. Este algoritmo se fundamenta en la división en segmentos cortos para realizar la evaluación de la transformada de Fourier (Oppenheim, Willsky, \& Nawab, 1998). 
Para realizar el algoritmo del cálculo de la transformada discreta de Fourier o DTF y poder obtener las potencias de las componentes espectrales, se utilizaron las siguientes ecuaciones:

$$
X(j w)=\sum_{n=0}^{L-1} x(n) * e^{-j w n}
$$

Ec. 5

Como se puede apreciar en la anterior ecuación, la potencia de una frecuencia $\mathrm{w}$ se puede evaluar en un fragmento de señal de longitud L, de igual manera se puede notar que los valores resultantes de la función X (jw) son números complejos, por esta razón los análisis de Fourier se hacen, por excelencia, en términos de la magnitud de estos números complejos (Manolakis \& Proakis, 1998). La ecuación 6, en términos de un número complejo rectangular, es la siguiente:

$$
X(j w)=\sum_{n=0}^{L-1} x(n)[\cos (w n)-j \operatorname{sen}(w n)]
$$

Ec. 6

De esta forma la relación $X$ (jw) puede ser pasada a la magnitud de sí misma como X (jw), y su relación matemática es la siguiente:

$|X(j w)|=\sqrt{\left[\sum_{n=0}^{L-1} x(n) \cos (w n)\right]^{\wedge} 2+\left[\sum_{n=0}^{L-1} x(n) \operatorname{sen}(w n)\right]^{\wedge} 2}$

Ec. 7

Esta ecuación matemática permite evaluar la potencia, o la intensidad de la componente $\mathrm{w}$ dentro de la muestra x(n), de longitud L. (Oppenheim, Willsky, \& Nawab, 1998).
El algoritmo de la DFT se trabajó a 256 puntos y se calcularon veinte componentes espectrales de cada señal.

\section{Resultados}

Figura 4. Prototipo final del ACRE

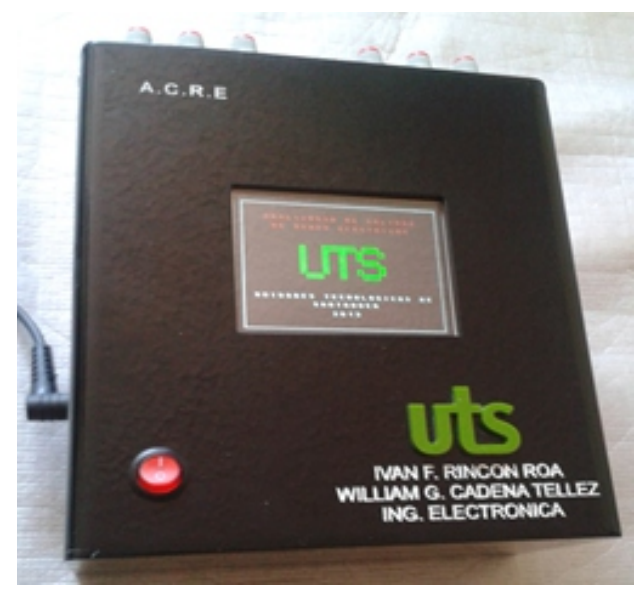

Fuente: Los autores

El hardware, el software y la interfaz de usuario del prototipo final del analizador de calidad de redes eléctricas "ACRE" fue probado con un analizador de calidad de energía eléctrica trifásica modelo 3945 de AEMC (AEMC Instruments, 2012), y las medidas de voltaje, corriente, desfases, potencias, armónicos, THD y frecuencia fueron comparadas directamente con este equipo patrón para verificar su correcto funcionamiento.

\section{Validación con el equipo patrón}

El equipo utilizado fue un analizador de calidad eléctrica modelo 3945 de AEMC, que se muestra en la Figura 5, junto con sus características de exactitud. 
Figura 5. Analizador de calidad eléctrica

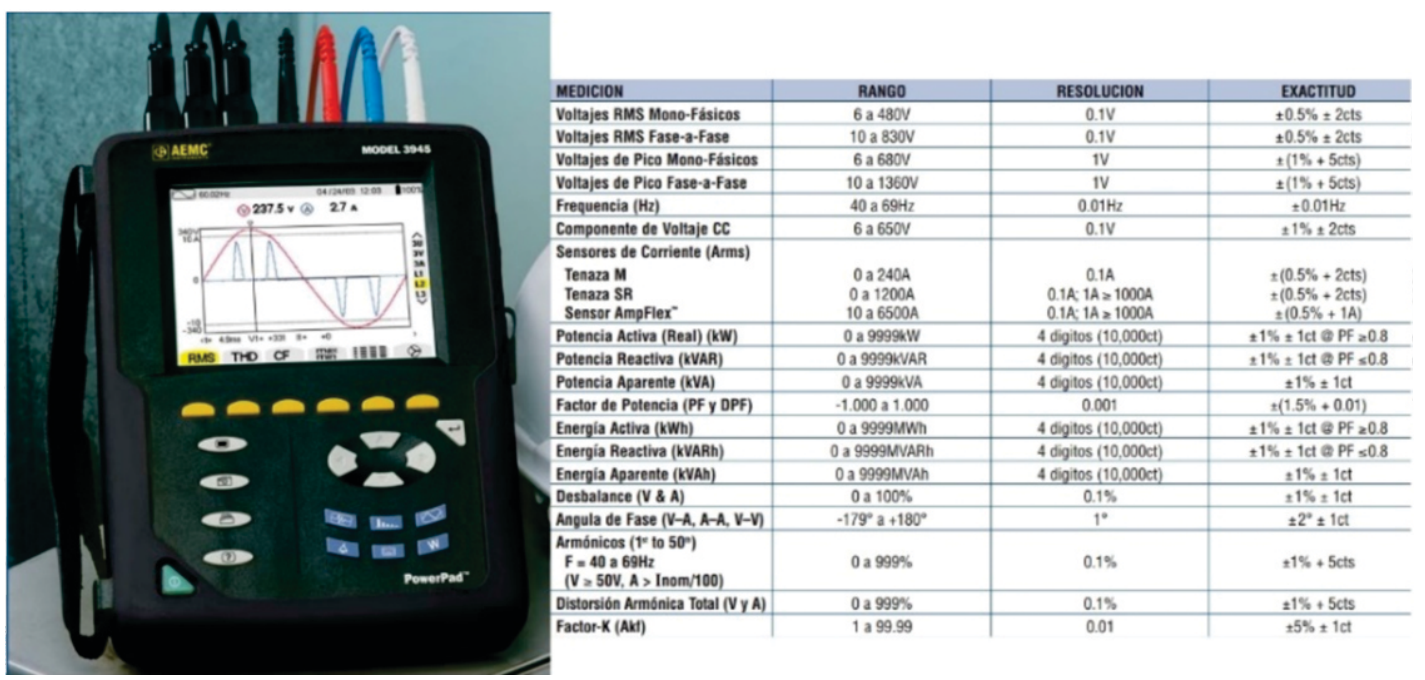

Fuente: Los autores

Se realizaron una serie de pruebas, a diferentes horas, en la red trifásica de la empresa Coomultrasan Multiactiva, para comparar directamente el equipo patrón con el prototipo final ACRE. Se verificó su correcto funcionamiento y se evaluaraon los porcentajes de error. En la Figura 6 puede evidenciarse el montaje realizado para la evaluación del equipo y en las figuras 7, 8,9 y 10 algunas de las comparaciones realizadas; la imagen de la izquierda es la medida del equipo ACRE y las imágenes de la derecha fueron hechas con el patrón.
Figura 6. Pruebas con el ACRE respecto AEMC 3945 en la red trifásica de Coomultrasan Multiactiva

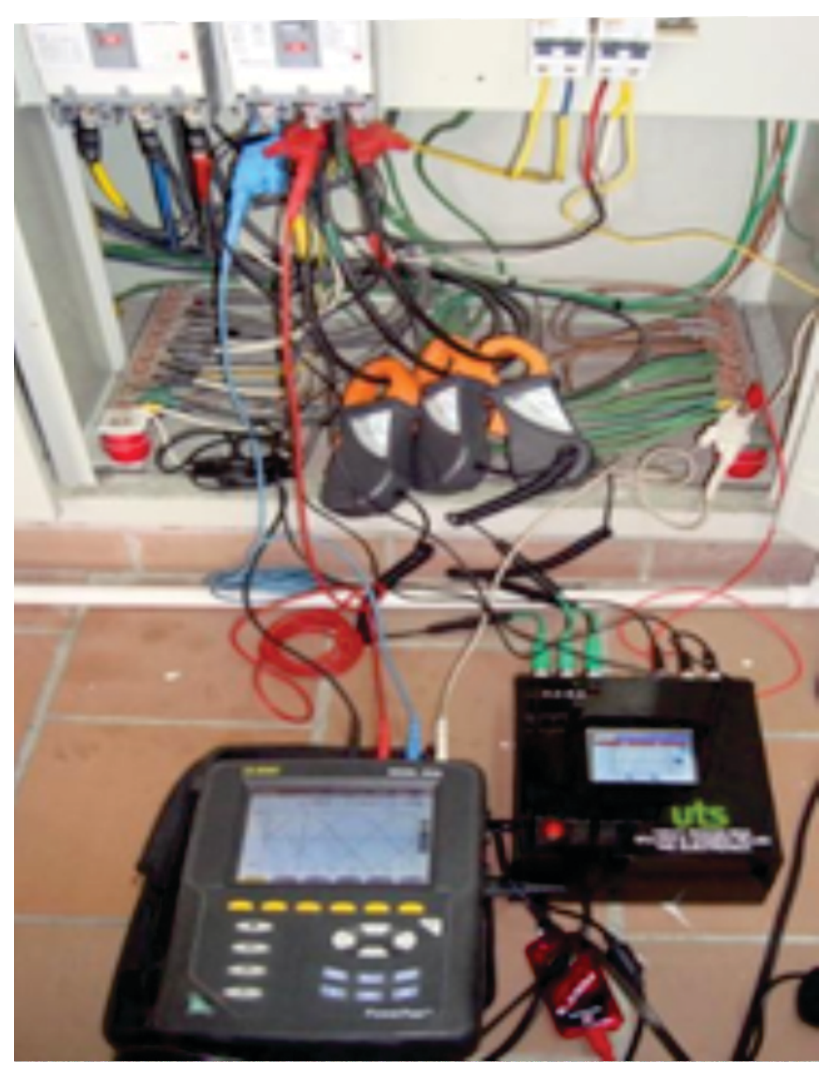

Fuente: Los autores 
IVÁN FERNANDO RINCÓN ROA, WILLIAM GUSTAVO CADENA TÉLLEZ, MARCO FIDEL FLÓREZ FRANCO Diseño y construcción de un analizador de redes eléctricas para sistemas trifásicos de bajo voltaje.

Figura 7. Señales de voltaje de fase

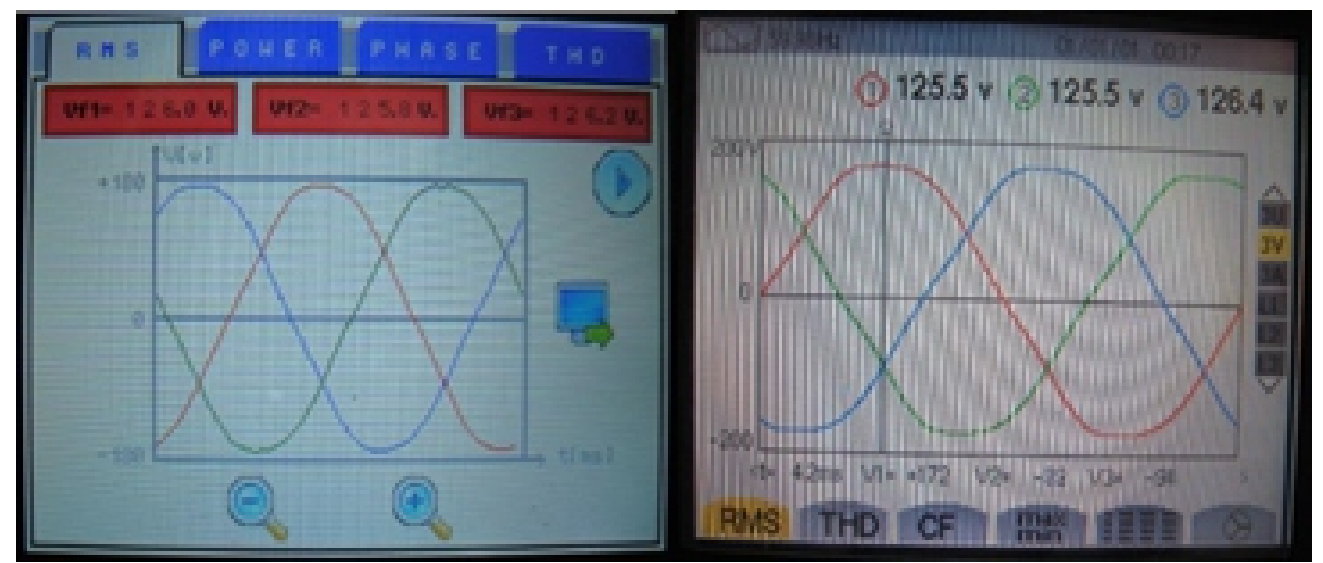

Fuente: Los autores

Figura 8. Señales de voltaje de línea

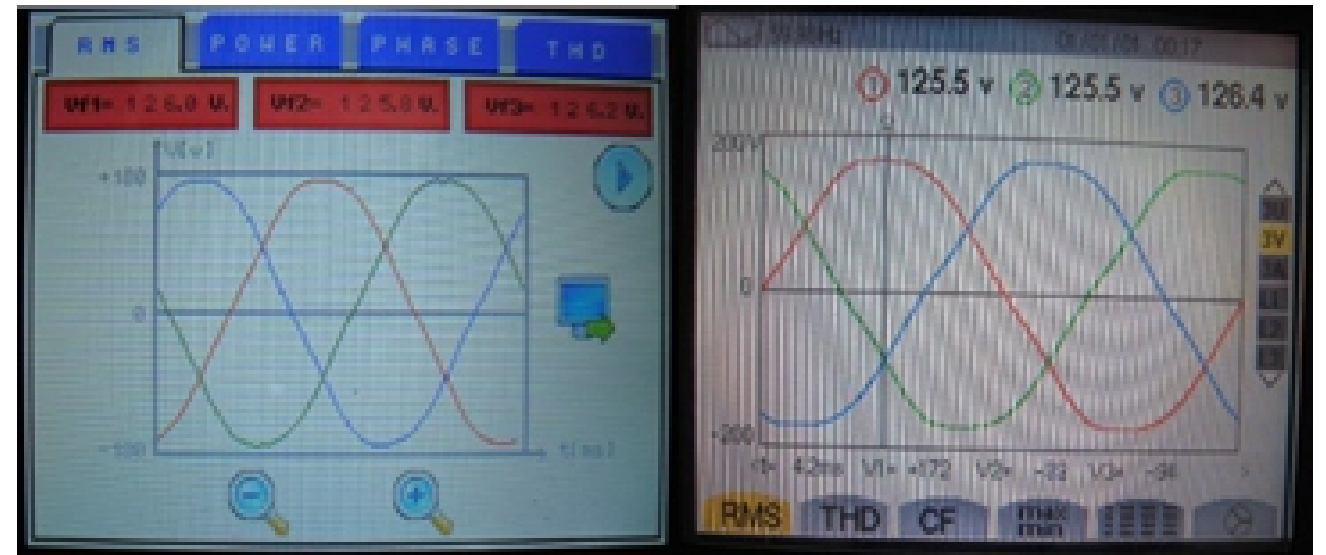

Fuente: Los autores

Figura 9. Potencias por fase

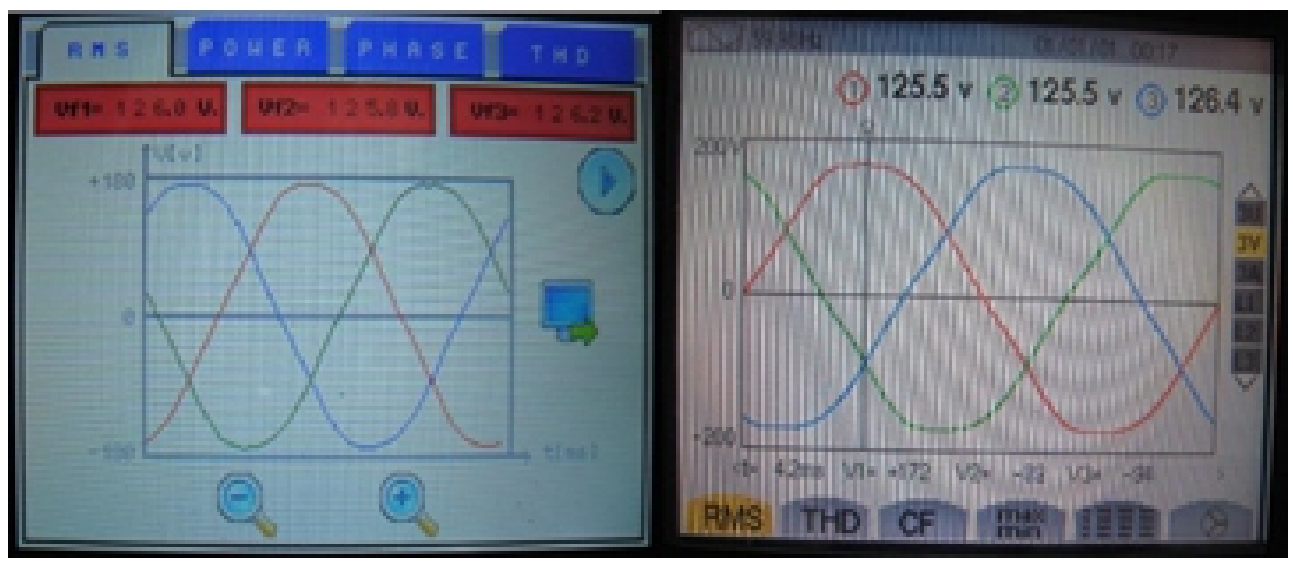

Fuente: Los autores 
Figura 10. Armónicos y THD de corriente de fase

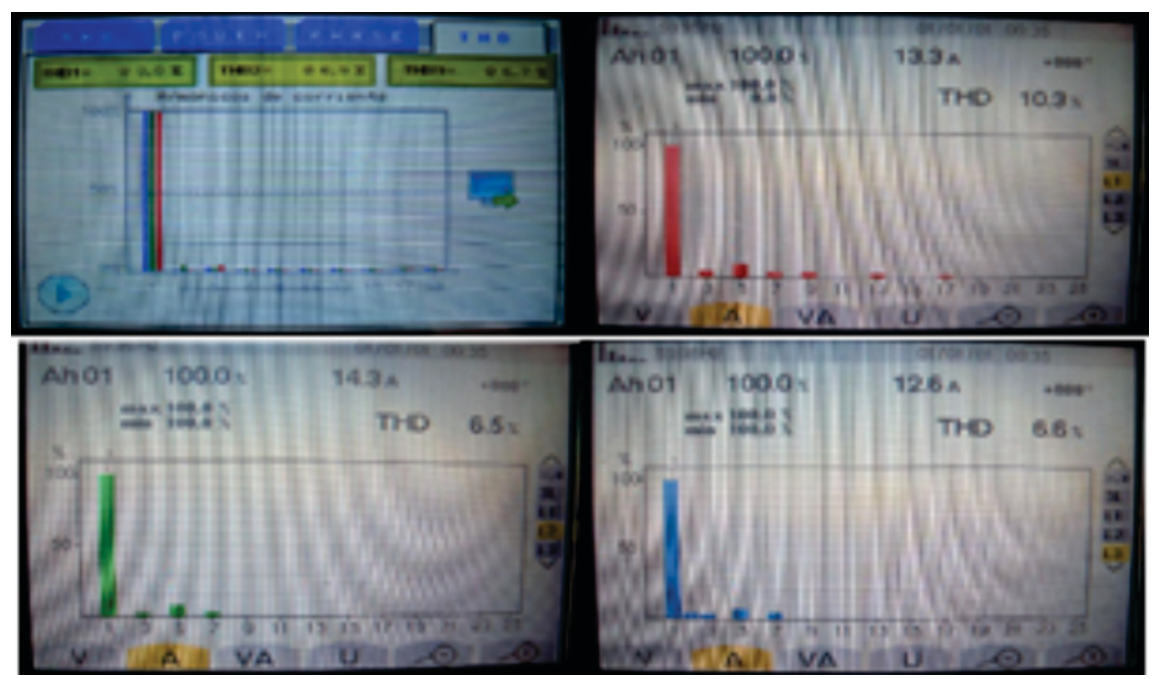

Fuente: Los autores

Los resultados de las pruebas y comparaciones con el equipo patrón se muestran en las tablas 1 a 3 . A partir de los errores arrojados en estas pruebas se puede concluir que el equipo es fiable en la medición de todos sus parámetros.

Tabla 1. Errores de las pruebas de la pantalla RMS

\begin{tabular}{lccc}
\hline & $\begin{array}{l}\text { Porcentaje de } \\
\text { error }- \text { Vf }\end{array}$ & $\begin{array}{l}\text { Porcentaje de } \\
\text { error - VL }\end{array}$ & $\begin{array}{l}\text { Porcentaje de } \\
\text { error - If }\end{array}$ \\
\hline $\mathbf{R}$ & 0.409 & 0.902 & 1.401 \\
$\mathbf{S}$ & 0.804 & 0.184 & 1.545 \\
$\mathbf{T}$ & 0.723 & 0.546 & 2.325 \\
\hline
\end{tabular}

Tabla 2. Errores de las pruebas de la pantalla power.

\begin{tabular}{|c|c|c|c|c|}
\hline & $\begin{array}{l}\text { Porcentaje de } \\
\text { e r o r } \\
\text { p o t e n i a } \\
\text { activa }(k W)\end{array}$ & $\begin{array}{l}\text { Porcentaje de } \\
\text { error potencia } \\
\text { reactiva } \\
(\mathrm{kVAR})\end{array}$ & $\begin{array}{l}\text { Porcentaje de } \\
\text { error potencia } \\
\text { aparente } \\
(k V A)\end{array}$ & $\begin{array}{c}\text { Porcentaje de } \\
\text { error factor de } \\
\text { potencia } \\
\text { Porcentaje }\end{array}$ \\
\hline L1 & 25.070 & 17.134 & 0.920 & 1.9684 \\
\hline $\mathbf{L} 2$ & 25.866 & 6.240 & 1.935 & 2.640 \\
\hline $\mathbf{L 3}$ & 23.477 & 8.338 & 1.592 & 4.813 \\
\hline
\end{tabular}


Tabla 3. Errores de la prueba de la pantalla THD

\begin{tabular}{cccc}
\hline & $\begin{array}{c}\text { Porcentaje de } \\
\text { error }- \text { Vf }\end{array}$ & $\begin{array}{c}\text { Porcentaje de } \\
\text { error }- \text { VL }\end{array}$ & $\begin{array}{c}\text { Porcentaje de } \\
\text { error - If }\end{array}$ \\
\hline $\mathbf{R}$ & 15.369 & 8.800 & 7.192 \\
$\mathbf{S}$ & 17.446 & 19.666 & 25.858 \\
$\mathbf{T}$ & 11.686 & 23.187 & 15.166 \\
\hline
\end{tabular}

\section{Comparación de los resultados con equipo equivalente}

En el mercado existen equipos comerciales que realizan funciones equivalentes al analizador de calidad de redes eléctricas ACRE UTS, como el producido por la empresa AEMC, cuyo modelo 3945 tiene un costo, en Colombia, de $\$ 10.800 .000$; el modelo analizador de redes trifásicas 434-II, Fluke, que cuesta $\$ 18.700 .000$, y el analizador de redes eléctricas Powerlogic PM8000 de Schneider Electric, que tiene un costo aproximado de $\$ 9.500 .000$. En comparación con estos equipos el costo para la fabricación en masa del ACRE UTS sería de tan solo $\$ 1.000 .000$ y podría comercializase en alrededor de $\$ 2.000 .000$, precio asequible para la mediana industria colombiana.

\section{Conclusiones}

Trabajar con integrados de montaje superficial SOIC, TQFP y SOT-223 produjo resultados notables en cuanto a la exactitud, ya que el error máximo fue del $4 \%$ comparado con el equipo patrón, además tuvo un excelente comportamiento frente al ruido y temperatura, pues la tecnología se ha perfeccionado tanto que su comportamiento se acerca mucho al esperado teóricamente, esto en comparación con los montajes realizados con tecnología DIP, que no son tan fieles al diseño.

Utilizar el transductor pinza de AC HT 4003 para sensar corriente fue una buena solución al problema de la adquisición de señal, pues la tensión de salida presentó un comportamiento lineal y en fase con la forma de onda de la señal de corriente que se sensaba, sin necesidad de introducir más hardware que degrade o distorsione la señal.

La implementación de una pantalla touchscreen es de gran utilidad, ya que se pueden visualizar los resultados de manera independiente del uso de un computador, obteniéndose de forma inmediata, sin necesidad de esperar a descargar los datos en un computador, además, se ahorra una interfaz para la utilización de los periféricos de entrada.

Para mejorar la funcionalidad de la pantalla touchscreen fue necesario calibrarla para determinar de forma correcta los puntos de toque.

Al finalizar el proyecto se puede decir que las ventajas más importantes de utilizar el dsPIC fueron la posibilidad de trabajar con un ADC de 32 canales de entrada, 2 módulos de ADC -los cuales pueden trabajar a una resolución de 12 bits 
o 10 bits- y 2 módulos de comunicación SPI de 16 bits.

Haber tenido en cuenta el diseño del PCB de las tarjetas electrónicas y la carcasa del equipo para la distribución de los conectores o periféricos de entrada y salida, facilitó el ensamble y la operación del equipo final.

De acuerdo con los errores hallados se puede decir que es mejor sensar tensión que corriente en los pines de entrada de los circuitos, pues medir tensión es más preciso. Cabe destacar que el HT 4003 sensa corriente de niveles de tensión aproximados de $1 \mathrm{mVrms} / \mathrm{A}$, mientras que el divisor de tensión sensa aproximadamente a $5.24 \mathrm{mVrms} / \mathrm{V}$, lo que permite que las señales de tensión que entran a la etapa de adecuación de señales de voltaje tengan una mejor relación de señal-ruido.

La respuesta de la etapa de adquisición de señal estuvo de acuerdo con los resultados que se esperaban, pues tuvo un comportamiento muy similar a los teóricos y simulados; esto se puede entender por el hecho de trabajar con elementos de montaje superficial y de alta gama, que tienen una tolerancia mínima y cuyas características se acercan a las ideales.

\section{Recomendaciones}

Se puede mejorar este prototipo implementando una memoria de almacenamiento masivo de datos SD, de modo que se puedan almacenar los datos de forma continua y puedan ser analizados fuera de línea y así observar el comportamiento de la red para largos periodos de tiempo.
Diseñar e implementar un software para PC que permita realizar análisis del comportamiento de las redes eléctricas trifásicas en grandes periodos de tiempo, y que acompañe los resultados del equipo.

Realizar un software de comunicación y visualización inalámbrica en tiempo real que le brinde al equipo la prestaciones necesarias para el monitoreo remoto de las redes.

\section{Referencias}

AEMC Instruments. (s.f.). Recuperado el 06 de 09 de 2012, de Analizador de calidad de energía eléctrica trifásica. Modelo 3945.: http:/www.gzingenieria.com/pdf/aemc3945.pdf

Carrasco, J. A. (s.f.). Circuitos Monofásicos y Trifásicos de Corriente Alterna. Recuperado el 09 de 08 de 2011, de Circuitos Monofásicos y Trifásicos de Corriente Alterna: http://metis.umh.es/jacarrasco/docencia/ep/Tem a1/1ph3ph.pdf.

Carrasco, J. (s.f.). Así funciona. La corriente alterna (C.A.). Recuperado el 11 de 08 de 2011, de Así funciona. La corriente alterna (C.A.).: http://www.asifunciona.com/electrotecnia/ke_c orriente_alterna/ke_corriente_alterna_1.htm.

Clavijo, J. (2011). Diseño y simulación de sistemas microcontrolados en lenguaje C (Vol. 10). Colombia: jrclavijo ISBN: 978-958-448619-6. 
Escobar, V., \& Jácome, P. (2009). Construcción e implementación de un prototipo terminal de consulta del Sistema de Administración Estudiantil (SAEw) mediante la utilización de una pantalla táctil. Ecuador: Facultad de Ingenieria Eléctrica y Electrónica.

Gómez, J. (2011). Instrumentación electrónica. Recuperado el 24 de 02 de 2012, de Instrumentación e lectróni c a: h t t p : / / w w w 2 . u c a. e s/g r u p invest/instrument_electro/ppjjgdr/Electronics_I nstrum/Electronics_Instrum_Files/temas/T13_d esplin.PDF.

Hayt, W., \& Kemmerly, J. (2005). Análisis de circuitos eléctricos.

Herrera, S., \& Flórez, J. (2004). Diseño y construcción de un prototipo de un medidor de armónicos de corriente basado en un procesador de señales digitales (DSP). Bucaramanga.

HT Instruments. (s.f.). Recuperado el 15 de 08 de 2011, de Accesorios de medida para instrumentos de verificación: http://www.htinstruments.com/transductor-decorriente-hasta-400aca

Manolakis, P., \& Proakis, J. (1998). Tratamiento digital de señales (3 ed.). Madrid, España: Pearson Prentice Hall.

Microchip Inc. (s.f.). Recuperado el 28 de 03 de 2012, de DSPIC33FJ256GP710 Datasheet.: http://ww1.microchip.com/downloads/en/Devic eDoc/70286C.pdf.
Microchip Inc. (2009). Recuperado el 26 de 05 de 2012, de MCP3909 / dsPIC33FJ128GP206 3-Phase Energy Meter Reference Design: http://ww1.microchip.com/downloads/en/Devic eDoc/51723a.pdf

Mornsun. (s.f.). Recuperado el 08 de 04 de 2012, de VFA_MP-6W \& VFB_MP-6W Series, Conversor D C - D C Dat a sheet: http://pdf1.alldatasheet.com/datasheetpdf/view/275117/MORNSUN/VFA1205MP6W.html

National Semicondutor Corporation. LM2937IMPX-15 Datasheet. (s.f.). Recuperado el 06 de 04 de 2012, de National Semicondutor Corporation. LM2937IMPX-15 Datasheet.: http://www.alldatasheet.com/data sheetpdf/pdf/8846/NSC/LM2937IMPX-15.html.

Oppenheim, A., Willsky, A., \& Nawab, S. (1998). Señales y sistemas. Pearson Educación.

Rojas, J., \& Reyes, E. (2011). Diseño y construcción del prototipo electrocardiógrafo portátil ECG_UTS02. Unidades Tecnológicas de Santander en convenio con la Universidad Distrital Francisco José de Caldas. Bucaramanga, Colombia.

Scribd. (s.f.). Recuperado el 18 de 09 de 2011, de Fundamentos de Armónicas en sistemas

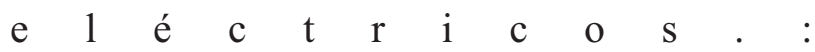
http://es.scribd.com/doc/4852712/Fundamentos - d e - A r m o n i c a s - e n s i s t e m a s electricos\#download 
Texas Instruments Inc. (s.f.). Recuperado el 05 de 04 de 2012, de TL084 Datasheet: http://www.ti.com/lit/ds/symlink/t1084.pdf.

Texas Instruments Inc. UA78M08C Datasheet. (s.f.). Recuperado el 05 de 04 de 2012, de Texas Instruments Inc. UA78M08C Datasheet.:

http://www.ti.com/product/ua78m08.

Texas Instruments Inc. UA78M33C Datasheet. . (s.f.). Recuperado el 05 de 04 de 2012, de Texas Instruments Inc. UA78M33C $\mathrm{D}$ a $\mathrm{t}$ a $\mathrm{s} \mathrm{h}$ e e $\mathrm{t}$. http://www.ti.com/product/ua78m33.
Zhou, X. L. (2012). Harmonic impacts of inverter-based distributed generations in low vVoltage distribution network. In Power Electronics for Distributed Generation Systems (PEDG). 2012 3rd IEEE International Symposium on. (pp. 615-620). IEEE. 